\title{
REVIEW
}

\section{Malaria in pregnancy}

\author{
JESUS R. ALVAREZ, ABDULLA AL-KHAN \& JOSEPH J. APUZZIO \\ Department of Obstetrics and Gynecology, Division of Maternal Fetal Medicine, UMDNF-New fersey Medical School, \\ Newark, NF, USA
}

\begin{abstract}
Recently, there has been a resurgence of malaria in densely populated areas of the United States secondary to human migration from endemic areas where factors such as cessation of vector control, vector resistance to insecticides, disease resistance to drugs, environmental changes, political instability, and indifference, have played a role for malaria becoming an overwhelming infection of these tropical underdeveloped countries. It is important for health care providers of gravida to be alert of the disease and its effects on pregnancy.
\end{abstract}

Keywords: Plasmodium, infection, outbreaks, antimalarial medication

\section{Introduction}

In the US, malaria was endemic until the beginning of the twentieth century with more than 500,000 cases per year. Malaria has a worldwide distribution with an incidence of 300-500 million cases per year with over 1-3 million deaths from the disease annually $[1,2]$. Despite a worldwide effort to curb the disease through vector control and treatment of the disease, resurgence has developed due to Plasmodium falciparum resistance.

Because of today's ease of travel and continental migration throughout the world, malaria has caused occasional outbreaks in densely populated areas of the United States [3-5]. There are 60 new cases reported in Florida annually [6].

It is important to recognize that pregnant patients at risk for malaria require immediate treatment. The effect of malaria on the gravida and the fetus is potentially severe and life-threatening, thus warranting a thorough understanding of its complications and treatments.

\section{Pathogenesis}

Malaria is an infection usually transmitted by the bite of an infected female Anopheles mosquito. The new parasite life cycle begins when parasites of the family
Plasmodia enter the bloodstream after the mosquito bite.

The four species of Plasmodium known to infect humans are (in decreasing order of prevalence): Plasmodium falciparum, $P$. vivax, $P$. malariae, and $P$. ovale. Due to the similarity of symptoms, it is virtually impossible to differentiate between the species without examining a microscopic peripheral blood smear. After a bite by an infected female Anopheles mosquito, sporozoites are released from the salivary gland of the mosquito and find their way to the human circulatory system starting the new parasite life cycle (Figure 1). These sporozoites travel to the liver invading hepatocytes to become the mature tissue schizonts. At this stage of the $P$. vivax and $P$. ovale life cycle, some of these schizonts become dormant hypnozoites. Inside the hepatocyte, an estimated 10,000 to 30,000 merozoites are produced per each infected hepatocyte. Merozoites are then released from the hepatocyte, each infecting a red blood cell (RBC). Inside the RBC, the parasite matures into a trophozoite, a schizont, and then into an asexual form for replication which returns back into the merozoite form. After 48-72 hours (depending on the species), approximately $25-30$ merozoites are released causing fever and symptoms. Inside the RBC, some Plasmodia may convert to a sexual form called a gametocyte. When a new female Anopheles mosquito bites an infected human host, released

Correspondence: Joseph J. Apuzzio, MD, UMDNJ-New Jersey Medical School, 185 South Orange Avenue, MSB E-506, P.O. Box 1709, Newark, New Jersey 07101-1709, USA. Tel: 973972 5557. Fax: 973972 4574. E-mail: susomd@yahoo.com 


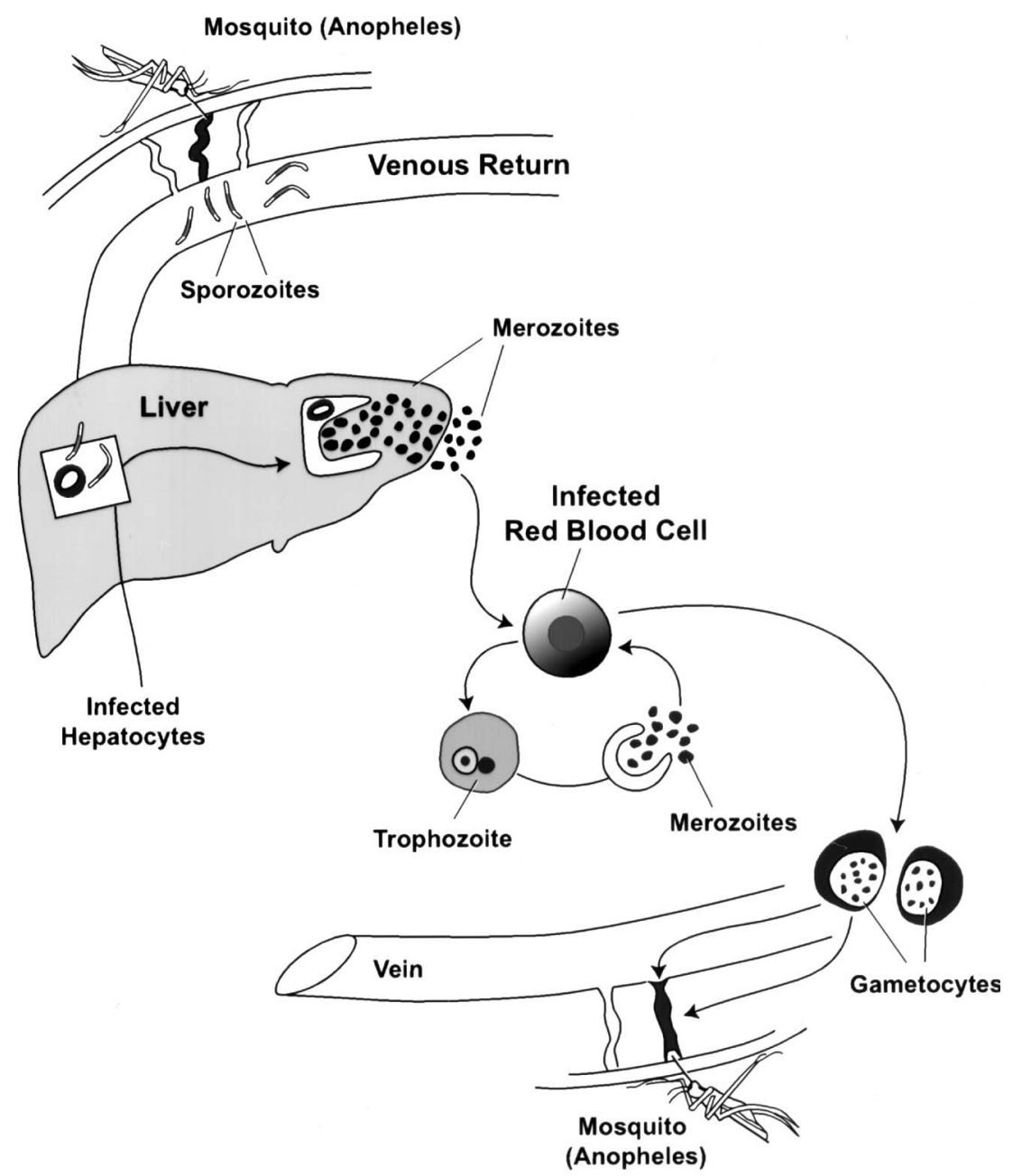

Figure 1. Life cycle of malaria parasite. An infected Anopheles mosquito bites and infects a human host starting its new malaria life cycle. The cycle is completed when an uninfected mosquito bites an infected human host, becoming infected by the transmission of gametocytes which then matures into sporozites inside the mosquito.

gametocytes from red blood cells, are taken by the mosquito. The gametocytes develop into either a male or female gametocyte within the gut. Fertilization occurs between male and female gametocytes, producing a diploid zygote. This zygote develops into an ookinete that invades the gut of the mosquito and becomes an oocyst. The oocyst undergoes meiosis producing haploid sporozoites that migrate to the salivary gland of the mosquito. The cycle is completed when this infected mosquito bites an unfortunate host.

Plasmodia, in the sporozoite form, enters the hepatocyte by binding to the circumsporozoite protein (CSP) [7]. The presence of the Duffy antigen has been demonstrated to be necessary for parasitic invasion of RBC's by $P$. vivax [8]. In P. falciparum, several molecules on the surface of endothelial cells act as receptors for infected RBCs [9-11].

$P$. falciparum infection causes cytoadherence of affected red blood cells in the microvasculature of the circulatory system which potentiates micro- thrombi formation leading to microvascular disease. The microthrombi affect a number of organs causing various symptoms and pathologies.

In addition to the cytoadherence of RBCs to endothelial cells in the microcirculation, the size, shape, and pliability of the infected RBC changes. The normal, 7-8 $\mu \mathrm{m}$ donut-shaped RBC, changes into a smaller, less deformable $4-5 \mu \mathrm{m} \mathrm{RBC}$. This can also potentiate microthrombus formation.

The human host responds by producing cytokines, interleukins, and tumor necrosis factor (TNF). The fever caused by malaria is secondary to a release or exposure to glycosyl phosphatidylinositol (GPI) from the RBC surface during the time of RBC rupture and merozoite release. As a result of this process, TNF- $\alpha$ is then released from macrophages resulting in fever $[12,13]$.

Each species of Plasmodium has a preference for RBC's of specific age. This selectivity limits their potential for reproduction and thereby the severity and duration of infection. Plasmodium falciparum has 
no preference and is considered the most serious form of the disease as it is capable of living in RBCs of all ages. As a result, a higher level of parasitemia can occur causing a more severe disease.

Sickle-cell trait has been shown to protect against severe malarial disease. Sequestration in the microvasculature where the oxygen tension is low inhibits parasite growth in falciparum malaria, but not in other Plasmodium infections. In addition to hemoglobin $\mathrm{S}$, hemoglobin $\mathrm{C}$ has been thought to be protective [14]. More recently, HLA-Bw53 and HLA-DRB1*1302 have been found to be protective genes in specific populations protecting against severe malaria $[15,16]$.

\section{Clinical findings of active disease}

Malaria is a multi-organ disease. A relationship between the level of parasitemia and risk of death has been demonstrated [17]. Malaria tends to be more severe in pregnant women, especially in nonimmune patients.

Malaria causes acute febrile illness with characteristic febrile paroxysms every $48-72$ hours. Depending on the general health of the patient and the species of the parasite, the severity and course of the attack will vary. Malaria often presents in 3 stagescold, hot and sweating stages. The cold stage begins with a subjective feeling of cold that progresses to severe shivering with a rapid thready pulse. The duration of this stage lasts $15-60$ minutes. Next, the hot stage begins as the patient develops a fever that can reach $104-106^{\circ} \mathrm{F}$. The skin is flushed and dry with a rapid, full and bounding pulse lasting for 26 hours. Finally, the sweating stage begins when the temperature falls and the patient begins to sweat profusely. In this stage the patient may become dehydrated. The sweating stage lasts $2-4$ hours. After resolution of the three stages, the patient may feel better for a short time before the cycle repeats itself. The time interval between cycles depends on the infecting parasite. $P$. vivax and $P$. ovale cycle every 48 hours (tertian malaria), $P$. malariae cycles every 72 hours (quartan malaria). $P$. falciparum cycles have asynchronous intervals usually within 36-48 hours. If untreated, these cycles continue from a week to longer than a month [18].

$P$. ovale and $P$. vivax are capable of remaining dormant in the liver in the form of hypnozoites and usually reappear in about 6-11 months causing a relapse. $P$. malariae, on the other hand, may persist at low levels in the circulation for up to 30 years without liver involvement.

$P$. falciparum is considered an acute form of malaria, usually with severe symptoms, and does not relapse. Falciparum malaria can cause chronic splenomegaly, hemolytic anemia, nausea and vomiting, diarrhea, lactic acidosis, hypoglycemia, jaundice, cerebral malaria, acute renal failure, severe anemia, and rarely pulmonary edema.

Plasmodium vivax and $P$. ovale are usually described together because they produce hypnozoites, cycle every 48 hours, and have similar clinical presentations. The hypnozoites can remain dormant in the liver for 6-11 months before becoming active and producing a relapse. Splenic rupture 2-3 months after initial infection can occur. No microvascular complications occur as with falciparum malaria. In addition, parasitemia is usually less severe compared with $P$. falciparum as only reticulocytes are affected [19]. Furthermore, $P$ falciparum suppresses hematopoiesis, whereas $P$. vivax and $P$. ovale have been found to stimulate hematopoiesis.

Laboratory findings typically seen in malaria include acute anemia, increased $\mathrm{LDH}$, reticulocytosis, and thrombocytopenia. In addition, proteinuria, hemoglobinuria, elevated serum creatinine, hyperbilirubinemia, elevated transaminases, lactic acidosis, and hypoglycemia may be seen.

\section{Screening and diagnosis}

The diagnosis of malaria during pregnancy requires laboratory confirmation, usually by blood film from a fingerstick. Light microscopy of Giemsa-stained blood smears with oil-immersion is necessary for specific parasite identification. $P$. vivax and $P$. ovale types of malaria have the characteristic Schuffner's dots allowing for easy identification. The blood film offers a cheap, easy and effective means of diagnosing malaria and the type of Plasmodium species causing the infection. However, $5-7 \%$ of cases are due to simultaneous infection with more than one species.

Recently, new tests to diagnose malaria have emerged. New dipstick tests (ICT-Malaria Pf, OptiMALr and the Kat-Quick kits) can detect histidine rich protein-2 (HRP-2) and/or parasite specific lactate dehydrogenase (pLDH) in Plasmodium falciparum infections. Studies have shown that these new tests have sensitivities approaching $100 \%$ [20] and show positive results up to 2 weeks after successful treatment of malaria.

Another new method is the polymerase chain reaction (PCR). With the use of PCR, it is possible to detect $<10$ parasites $/ 10 \mu \mathrm{L}$ of blood. Zalis et al. reported a PCR detection rate by amplification using ethidium bromide staining of the p126 $P$. falciparum gene of .005 parasites $/ \mu \mathrm{L}$ without radioactive material [21]. However, this method is much more expensive than a blood film and is not readily available in many areas involved in the epidemic. 
Immunodiagnosis using ELISA testing is useful, nevertheless malaria may persist for months after the infection. For this reason, ELISA testing is more useful for epidemiological purposes.

\section{Malaria in pregnancy}

Women living in endemic areas are considered to have some immunity and malarial infections are usually asymptomatic or with less severe symptoms [22]. Conversely, the disease is almost always symptomatic, and potentially lethal, in non-immune patients particularly gravid females. This is thought to be due to higher levels of circulating immunosuppressive corticosteroids in pregnant women $[23,24]$. Preterm delivery, IUGR, abortion, stillbirth, eclampsia postpartum hemorrhage, puerperal fever, and maternal/fetal death are some complications associated with malaria particularly with $P$. falciparum. Women in their first trimester of pregnancy are at a high-risk of developing severe, life-threatening malaria-associated complications. Studies have shown that pregnant women attract at least twice as many mosquitoes as non-pregnant patients [25,26]. Risk factors for adverse outcomes of women infected with malaria during pregnancy include HIV + status, low parity, non-immune, suboptimal treatment, and pregnancy during hightransmission season [27].

When infection of the placenta occurs, an increased risk of stillbirth and prematurity has been noted [28]. The placenta becomes involved when infected, adhesive RBCs sequester in the intervillous space [2]. Deterioration in placental function may occur, causing transient fetal hypoxia. Arbeille et al. showed how the hypoxic index value during crisis can predict an abnormal fetal heart at delivery [29]. Primigravidas appear to be at the highest risk [30]. Is believed that in subsequent pregnancies, affected women are protected from sequestration of RBCs in part by antibodies that prevent cytoadhesion of infected RBCs in the placenta [2].

Approximately $8-14 \%$ of LBW babies are a result of preterm labor directly related to a malarial infection, and about 3-14\% of infant death is related to the disease [3]. Precipitous labor and uterine rupture have also been reported as complications of malarial infection [31]. The incidence of malaria may be higher in HIV-positive pregnant females according to a study in Rwanda [32].

Anemia and thrombocytopenia are commonly seen in all forms of malaria, but may be especially severe with $P$. falciparum. Severe anemia results in infected patients for two reasons. First is due to RBC destruction associated with release of merozoites. Secondly, the elevated TNF- $\alpha$ produced from falciparum malarial infection causes suppression of hematopoeisis. Thrombocytopenia also occurs more commonly in pregnant patients with malaria and can lead to prolonged and easy bleeding. Due to the potentially harmful effects of malaria to both the mother and the fetus in utero, it is therefore recommended that these patients receive chemoprophylaxis to prevent malarial infection.

Hypoglycemia and lactic acidosis due to malaria is seven times more frequent in pregnancy than in a non-pregnant woman [33]. Lactic acidosis and hypoglycemia result from hepatic involvement. Due to the metabolic requirements of both the fetus and the parasites, a pregnant woman may suffer from severe hypoglycemia. The Plasmodium parasites not only consume maternal glucose but also stimulate the pancreatic beta cells, leading to hyperinsulinemia, and ultimately severe hypoglycemia [34,35]. The hypoglycemia is manifested by dizziness, blurred vision, cold extremities, and hypotension. When severe enough, the hypoglycemia can progress to mental status changes and convulsions. Early recognition and treatment of patients with falciparum malaria and hypoglycemia can significantly affect the patient's ultimate outcome [36].

Cerebral malaria is a common presentation in pregnant patients. Particularly concerning is the increased case-fatality rate of cerebral malaria in pregnant patients of up to $50 \%$ [37]. Case-fatality rates is approximately $20 \%$, and $3 \%$ of patients develop permanent neurologic sequelae [37]. Within days to weeks a gravid patient may progress to confusion, impaired consciousness, agitation, coma, convulsions, shock, and death. The extent of dysfunction is reflective upon virulence, immune status of patient, delay in treatment, or a combination of the above. Patients surviving cerebral malaria may have persistent sequela including speech disorders, behavioral disorders, ataxia, hemiplegia, epilepsy, and blindness.

Several complicated cases reported from China described cerebral malaria with coma, high fever, convulsions, anemia, and splenomegaly. Eclampsia, blackwater fever, renal failure, cardiopulmonary failure, postpartum hemorrhage, and puerperal infection were reported as causes of death in many of the patients. Recommendation for early termination of pregnancy in patients with eclampsia was described as life-saving [38].

The oliguric acute renal failure associated with falciparum malaria is relatively common in nonimmune patients. Microvascular disease causes an occlusion in the renal vasculature thereby preventing exchange of glucose and oxygen in the kidney. Intravascular hemolysis allows free hemoglobin and malarial pigment to damage the kidneys. This is known as "blackwater fever". Dialysis for several weeks may be necessary for survival in patients with 
severe disease. This presents as dark or black urine and is caused by G6PD deficiency-related hemolysis from antimalarial drugs and/or directly from the malarial infection.

The main complication associated with $P$. malariae is immune complex glomerulonephritis (parasite antigen with host $\operatorname{IgG}$ ) and usually occurs 3-6 months after transmission [39]. No microvascular complications are seen with this form of malaria. $P$. malariae usually produces mild symptoms and produces acute illness only in immunocompromised patients. However, parasitemia may persist for up to 20-30 years.

Although uncommon, pulmonary edema can also result from microvascular compromise causing ARDS [35,39]. It is believed that TNF- $\alpha$ by itself can produce the severe pulmonary edema and ARDS observed in falciparum malaria [40]. Treatment with artificial ventilation may be life-saving.

\section{Antimalarial medications}

Aminoquinolones act by inhibiting heme polymerase the enzyme responsible for breakdown of the toxic hemoglobin in the food vacuole of the parasite [41]. As a result, the hemoglobin accumulates and lyses the cell membrane. Drugs in this class approved in the US include chloroquine, quinidine, hydroxychloroquine and the newer aminoquinolones, mefloquine and halofantrine. The drug regimen chosen for prophylaxis and treatment of malaria depends on the suspected species of Plasmodium parasite and the patient's ability to take medication.

Chloroquine, a Category $\mathrm{C}$ drug, has been the treatment of choice for almost 50 years especially in pregnancy. However, resistance of $P$. falciparum and $P$. vivax is becoming widespread in endemic areas. Chloroquine is used as a single first-line treatment in many areas for $P$. vivax, $P$. malariae, and $P$. ovale, or in combination with another drug. It may be given intravenously if the patient is unable to tolerate oral medications. Chloroquine must be given very carefully because cardiac arrhythmias may develop. Pregnancies exposed to chloroquine have not demonstrated increased rates of congenital abnormalities, stillbirth, or low birth weight and the drug is considered safe in the first trimester [42] and breastfeeding. Primiparous mothers have been shown to benefit from chloroquine prophylaxis in pregnancy by reducing the risk of anemia at birth [43]. In addition, chloroquine has been shown to inhibit release of $\mathrm{TNF}-\alpha[12,44]$.

Hydroxychloroquine is another aminoquinolone that is used mainly for prophylaxis. It is effective against all Plasmodia except chloroquine-resistant $P$. falciparum.
Quinine is effective in vivo against all strains of malaria, especially in chloroquine-resistant infections, but has a high gametocyte carriage rate. The combination of quinine with clindamycin has been used as a back-up for quinine/artesunate resistant $P$. falciparum [45]. High first dose quinine provides increased parasite clearance but is a known abortafacient at high doses and can cause transient partial hearing loss as well. However, pregnancies exposed to quinine have not shown an increased rate of congenital abnormalities, stillbirth, or low birthweight and the drug is considered safe in the first trimester [42]. Furthermore, quinine has been shown to prevent premature contractions and fever in pregnant women [46]. Quinine is not available in the US, but the antiarrthymic quinidine is substituted for quinine [47]. Quinidine is listed as a Category C drug and considered safe in breast-feeding patients.

Due to the high resistance rates of Plasmodium, both prophylaxis and treatment regimens are tailored to the specific region from which the patient was infected. Mefloquine is effective against all Plasmodia and has been shown to shorten the parasite clearance time compared with quinine in susceptible species [48]. It is relied upon in nearly every endemic area [37]. Mefloquine has been shown to be safe in the second and third trimesters, but there is not sufficient evidence to promote its use during the first trimester [22]. It is listed as Category C, has not been associated with abortion, low birth weight, mental retardation, or congenital abnormalities. However, an increased risk of stillbirth has been demonstrated [49]. If the parasite is resistant to mefloquine, halofantrine should not be used.

Another new drug that has been shown to be reliable in resistant species is halofantrine. It is not used prophylactically. It is effective against all sensitive Plasmodia in the asexual merozoite form. This drug should not be used in mefloquine-resistant infections as the mechanism of action is the same. Halofantrine can produce prolongation of the QT interval of an electrocardiogram, and may cause ventricular arrhythmias. This drug is listed as a category $\mathrm{C}$ but considered contraindicated in pregnant or lactating women.

Primaquine is used for treatment of hypnozoites in $P$. vivax and $P$. ovale infections. It is listed as Category $\mathrm{C}$ with an uncertain breast-feeding safety profile.

Proguanil is a new drug that is effective against early tissue schizonts of $P$. vivax infections. It may be used for prophylaxis of pregnant women and nonimmune patients at risk. It can be used with chloroquine for prophylaxis in areas of low prevalence of chloroquine resistance. The newly approved Malarone $^{\mathrm{TM}}$, a combination of proguanil and atovaquone, may be used to treat acute uncomplicated falciparum malaria. 
Amodiaquine is another aminoquinolone that is used outside the US for uncomplicated cases of malaria. It is similar to chloroquine and usually used in conjunction with artesunate. When used by travelers for prophlaxis, fatal adverse drug reactions have been reported [50,51].

Several derivatives of the Chinese herb, Qinghao$\mathrm{su}$, are used in the treatment of malaria outside the US. Artemesinin, artesunate, artemether, and arteether are some examples. These drugs are effective against all species of Plasmodia especially in chloroquine-resistant $P$. falciparum. Artesunate has been shown to shorten the parasite clearance time in susceptible patients compared with quinine [48]. Artesunate suppositories have been shown to be as effective as intravenous administration in treating $P$. falciparum $[52,53]$. The drug appears to be well tolerated with no evidence of adverse effects significantly different from controls [54].

Although pyrimethamine is primarily used to treat toxoplasmosis in the US, it may also be used to treat malaria. Pyrimethamine combined with chloroquine can be used to eradicate the latent hepatic phase of $P$. vivax and $P$. ovale infections. However, folic acid should be given to prevent deficiency. When possible, treatment with pyrimethamine should be delayed until after delivery of the fetus, because of the increased risk of hemolytic anemia in the fetus [13]. Even though resistance to pyrimethamine is common, dapsone combined with pyrimethamine is useful in resistant cases. It is listed as a Category C drug and considered safe during pregnancy.

Sulfadoxine is a sulfa drug not available in the US that is combined with pyrimethamine for the treatment of chloroquine-resistant $P$. falciparum. Fansidar ${ }^{\mathbb{R}}$, the combination of pyrimethamine and sulfadoxine, can cause potentially fatal StevensJohnson syndrome and is no longer used. Significant levels in the newborn can occur that may persist for days after delivery when given near term. This drug can cause jaundice, hemolytic anemia and potentially kernicterus in the newborn. Sulfa drugs are considered safe in breast-feeding and are listed as Category B.

Although not used for prophylaxis, teytracycline may be used in combination with other drugs for the treatment of malaria. Combined with quinine, tetracycline can be used against resistant $P$. falciparum. It is not used for prophylaxis. A longer-acting derivative of tetracycline, doxycycline, is also used in treatment of malaria. It is used in patients unable to tolerate mefloquine or reside in mefloquine-resistant areas. However, both are Category D drugs and are not recommended in pregnancy or breastfeeding mothers.

As a general rule, non-immune patients should be hospitalized for treatment to prevent and/or treat complications of falciparum malaria, especially when pregnant. Semi-immune patients can usually be treated as an outpatient for all species of malaria. For patients with high levels of parasitemia exceeding $15 \%$ and in addition to medications, exchange transfusion may be considered [55]. However, patients with parasitemias exceeding $50 \%$ have survived without exchange transfusion [56]. Treatment with corticosteroids was used for many years in the treatment of cerebral malaria but has been shown to be harmful as evidenced by longer duration of coma and worse outcomes [57]. Therefore, steroids should no longer be used in cerebral malaria.

\section{Congenital malaria}

Congenital malaria affects $7 \%$ of newborns in endemic areas. Malarial induced anemia in a pregnant patient is serious, because it can affect the oxygen delivery to the developing fetus. This anemia has been linked to infants born with low birth weight and even spontaneous abortions due to fetal hypoxia [34]. Steketee et al. estimated a yearly worldwide infant mortality of 75,000-200,000 directly related to malaria in pregnancy [58].

The level of parasitemia is proportional to the degree of anemia, thus a higher level of parasites in the blood results in a more profound anemia, hence a greater chance for fetal growth complications. It was also hypothesized that malarial infection of the placenta leads to a generalized placental thickening, thus hindering the passage of oxygen and nutrients and leading to fetal growth retardation [22].

In a study conducted by McGregor of Gambian pregnancies, it was discovered that the average birth weight of primagravidas infected with malaria was $150 \mathrm{~g}$ below normal, while there were no statistically significant difference seen in the birth weights of infants from multi-gravid females [59,22].

\section{Prevention}

In areas with a high prevalence of malarial parasitemia and anemia, prophylaxis with a drug proven to be effective for that particular area should be considered during pregnancy [60]. In fact, when drugs were given routinely for prophylaxis locally active for malaria the incidence of low birth weight and anemia was reduced. However, the effect appears to be limited to women with low parity $[43,61]$. Despite reports of resistance of $P$. falciparum to chloroquine, the benefit in reducing anemia at delivery was still noted.

Chemoprophylaxis should be started 2 weeks in advance to travel and should be continued for 4 weeks after return. Chloroquine is the agent of choice for prophylaxis and is considered safe for pregnant women and children. For chloroquineresistant areas, mefloquine should be used. In 
pregnancy, chemoprophylaxis is very important in preventing disease especially in primigravidas. Reports have indicated that mefloquine is safe [62].

Early studies involving the SPf66 vaccine demonstrated $10-30 \%$ effectiveness [63]. More recently, the SPf66 malaria vaccine has appeared to significantly reduce initial attacks of $P$. falciparum malaria according to a Cochrane collaborative review [64].

\section{Conclusions}

With the resurgence of malaria, the widespread harm caused by the disease and with the potential to spread beyond its current endemic areas, medical professionals must be vigilant to the prevention, recognition and treatment of malaria while being aware that malaria can have additional effects on the mother and fetus. Knowledge of this additional information will provide the health care provider with the best tools to provide the best opportunity for positive outcomes for mother, fetus and the community. For the most up-to-date information on malaria visit the "Roll Back Malaria" website [www.rbm.who.int]. There are many treatment regimens available, and the most up to date treatment protocols in the US can be obtained from the CDC's malaria hotline at (404) 332-4555 and on the CDC travel website [http://www.cdc.gov/travel].

Even though there are inherent risks associated with treatment of malaria, these risks are outweighed by the potential harm that may caused to the fetus in utero and the mother and can even result in death. Thus treatment of pregnant females with malaria is certainly indicated.

\section{References}

1. World Health Organization. WHO Expert Committee on Malaria: Twentieth report. 1998 Geneva Switzerland. World Health Organ Tech Rep Ser 2000;892:1-74.

2. Andrews KT, Lanzer M. Maternal malaria: Plasmodium falciparum sequestration in the placenta. Parasitol Res 2002; 88:715-723.

3. Brook JH, Genese CA, Bloland PB, et al. Malaria probably locally acquired in New Jersey. N Engl J Med 1994;331: 22-23.

4. Layton M, Parise ME, Campbell CC, et al. Malaria transmission in New York City, 1993. Lancet 1995;346: 729-731.

5. Anonymous. Local transmission of Plasmodium vivax malaria - Houston, Texas, 1994. MMWR 1995;44:295;301-303.

6. Center for Disease Control. Multifocal Autochthonous Transmission of Malaria - Florida 2003. MMWR 2004; 53(19):412-413

7. Cerami C, Frevert U, Sinnis P, et al. The basolateral domain of the hepatocyte plasma membrane bears receptors for the circumsporozoite protein of Plasmodium falciparum sporozoites. Cell 1992;70:1021-1033.

8. Miller LH, Mason SJ, Clyde DF, et al. The resistance factor to Plasmodium vivax in blacks. N Engl J Med 1976;295:302304.
9. Aikawa M, Iseki M, Barnwell JW, et al. The pathology of human cerebral malaria. Am J Trop Med Hyg 1990;43:30-37.

10. Chulay JD, Ockenhouse CF. Host receptors for malariainfected erythrocytes. Am J Trop Med Hyg 1990;43:6-14.

11. Ockenhouse CF, Tegoshi T, Maeon Y, et al. Human vascular endothelial cell adhesion receptors for Plasmodium falciparuminfected erythrocytes: Roles for endothelial leukocyte adhesion molecule 1 and vascular cell adhesion molecule 1. J Exp Med 1992;176:1183-1189.

12. Schofield L, Hackett F. Signal transduction in host cells by a glycophosphatidylinositol toxin of malaria parasites. J Exp Med 1993;177:145-153.

13. Kwiatkowski D, Canon JG, Manogue KR, et al. Tumor necrosis factor production in falciparum malaria and its association with schizont rupture. Clin Exp Immunol 1989;77:361-366.

14. Modiano D, Luoni G, Sirima BS, et al. Hemoglobin C protects against clinical Plasmodium falciparum malaria. Nature 2001;414:305-308.

15. Hill AVS. Malaria resistance genes: a natural selection. Trans R Soc Trop Med Hyg 1992;86:225-226.

16. Hill AVS. Genetic susceptibility to malaria and other infectious diseases: from the MHC to the whole genome. Parasitology 1996;112:75-84.

17. Field JW. Blood examination and prognosis in acute falciparum malaria. Trans R Soc Trop Med Hyg 1949;43:33-48.

18. Cot M, Roisin A, Barro D. Effect of chloroquine chemoprophylaxis during pregnancy on birth weight: Results of a randomized trial. Am J Trop Med Hyg 1992;46:21.

19. Neva FA. Observations on induced malaria: Looking back for a view of the future. Am J Trop Med Hyg 1977;26:211-216.

20. Palmer CJ, Lindo JF, Klaskala WI, et al. Evaluation of the OptiMAL $^{10}$ Test for Rapid Diagnosis of Plasmodium vivax falciparum malaria. Am J Trop Med Hyg 1999;60:173-176.

21. Zalis MG, Ferreira-da-Cruz MF, Balthazar-Guedes HC, et al. Malaria diagnosis: standardization of a polymerase chain reaction for the detection of Plasmodium falciparum parasites in individuals with low-grade parasitemia. Parasitol Res 1996; 82:612-616.

22. Silver HM. Malarial infection during pregnancy. Infect Dis Clin North Am 1997;11:99-107.

23. Rasheed F, Bulmer J, Dunn D. Suppressed peripheral and placental blood lymphoproliferative responses in first pregnancies: Relevance to malaria. Am J Trop Med Hyg 1993;48:154.

24. Vleugels MP, Eling WM, Rolland R, et al. Cortisol and loss of malaria immunity in human pregnancy. Br J Obstet Gynaecol 1987;94:758-764.

25. Ansell J, Hamilton KA, Pinder M, et al. Short-range attractiveness of pregnant women to Anopheles gambiae mosquitoes. Trans R Soc Trop Med Hyg 2002;96:113-116.

26. Lindsay S, Ansell J, Selman C, et al. Effect of pregnancy on exposure to malaria mosquitoes. Lancet 2000;355:1972.

27. Steketee RW, Wirima JJ, Campbell CC. Developing effective strategies for malaria prevention programs for pregnant African women. Am J Trop Med Hyg 1996;55:95-100.

28. Steketee RW. Malaria prevention in pregnancy: when will the prevention programme respond to the science. J Health Popul Nutr 2002;20:1-3.

29. Arbeille P, Caries G, Tobal N, et al. Fetal flow redistribution to the brain in response to malaria infection: does protection of the fetus against malaria develop over time? J Ultrasound Med 2002;21:739-746.

30. Alecrim WD, Espinosa FE, Alecrim MG. Plasmodium falciparum infection in the pregnant patient. Infect Dis Clin North Am 2000;14:83-95.

31. Bakri YN, Martan A, Amri A. Pregnancy complicated by malaria, precipitate labor and uterine rupture. Int J Gynaecol Obstet 1992;38:231-233. 
32. Ladner J, Leroy V, Simonon A, et al. The Pregnancy and HIV Study Group (EGE). HIV infection, malaria, and pregnancy: a prospective cohort study in Kigali, Rwanda. Am J Trop Med Hyg 2002;66:56-60.

33. Luxemburger C, Ricci F, Nosten F. The epidemiology of severe malaria in an area of low transmission in Thailand Trans R Soc Trop Med Hyg 1997;91:256-262.

34. Hubert, TV. Congenital Malaria in the United States: Report of a case and review. Clin Infect Dis 1992;14:922.

35. Warrell DA, Looareesuwan S, Warrell MJ, et al. Dexamethasone proves deleterious in cerebral malaria: A double-blind clinical trial in 100 comatose patients. N Engl J Med 1982; 306:313-318.

36. Kocher DK, Thanvi I, Kumawat BL, et al. Importance of blood glucose level at the time of admission in severe and complicated malaria. J Assoc Physicians India 1998;46:921-922.

37. White NJ. Malaria. In: Cook GC, editor. Manson's tropical diseases. 20th ed. London: WB Saunders; 1996. pp 1087-1164.

38. Ma C, Yussuf L. Clinical study on 24 cases of eclampsia with cerebral malaria. Zhonghua Fu Chan Ke Za Zhi 1999; 34:389-391.

39. Kibukamusoke JW, Hutt MSR, Wilks NE. The nephritic syndrome in Uganda and its association with quartan malaria. Q J Med 1967;36:393-408.

40. Tracey KJ, Fong Y, Hesse DG, et al. Anti-cachectin/TNF monoclonal antibodies prevent septic shock during lethal bacteremia. Nature 1987;330:662-664.

41. Slater AF, Cerami A. Inhibition by chloroquine of a novel haem polymerase enzyme activity in malaria trophozoite. Nature 1992;355:167-169.

42. McGready R, Thwai KL, Cho T, et al. The effects of quinine and chloroquine antimalarial treatments in the first trimester of pregnancy. Trans R Soc Trop Med Hyg 2002;96:180-184.

43. Salihu HM, Naik EG, Bosny JP, et al. Weekly chloroquine prophylaxis and the effect on maternal haemoglobin status at delivery. Trop Med Int Health 2002;7:29-34.

44. Tracey KJ, Fong Y, Hesse DG, et al. Anti-cachectin/TNF monoclonal antibodies prevent septic shock during lethal bacteremia. Nature 1987;330:662-664.

45. McGready R, Cho TA, Samuel VL, et al. Randomized comparison of quinine-clindamycin versus artesunate in the treatment of falciparum malaria in pregnancy. Trans $\mathrm{R}$ Soc Trop Med Hyg 2001;95:651-656.

46. Looareesuwan S, Phillips RE, White NJ, et al. Quinine in severe falciparum malaria in late pregnancy. Lancet 1985;2:4-8.

47. Center for Disease Control: Treatment of severe Plasmodium falciparum malaria with quinidine gluconate: Discontinuation of parenteral quinine from CDC drug service. MMWR 1991;40:240.

48. Bounyasong $S$. Randomized trial of artesunate and mefloquine in comparison with quinine sulfate to treat $P$. falciparum malaria pregnant women. J Med Assoc Thai 2001;84:12891299.
49. Nosten F, Vincenti M, Simpson J, et al. The effects of mefloquine treatment in pregnancy. Clin Infect Dis 1999;28:808-815.

50. Hatton CS, Peto TE, Bunch C, et al. Frequency of severe neutropenia associated with amodiaquine prophylaxis against malaria. Lancet 1986;22:411-414.

51. Neftel KA, Woodtly W, Schmid M, et al. Amodiaquine induced agranulocytosis and liver damage. $\mathrm{Br}$ Med J 1986;292:721-723.

52. Hien TT, Arnold K, Vinh H, et al. Comparison of artemisinin suppositories with intravenous artesunate and intravenous quinine in the treatment of cerebral malaria. Trans R Scop Trop Med Hyg 1992;86:582-583.

53. Arnold K, Tran TH, Nguyen TC, et al. A randomized comparative study of Artemesinin suppositories and oral quinine in acute falciparum malaria. Trans R Scop Trop Med Hyg 1990;84:499-502.

54. McGready R, Cho T, Keo NK, et al. Artemisinin antimalarials in pregnancy: a prospective treatment study of 539 episodes of multidrug-resistant Plasmodium falciparum. Clin Infect Dis 2001;33:2009-2016.

55. Miller KD, Greenberg AE, Campbell CC. Treatment of severe malaria in the United States with a continuous infusion of quinidine gluconate and exchange transfusion. $\mathrm{N}$ Engl J Med 1989;321:65-70.

56. Marik PE. Severe falciparum malaria: Survival without exchange transfusion. Am J Trop Med Hyg 1989;41:627629.

57. Warrell DA, Looareesuwan S, Warrell MJ, et al. Dexamethasone proves deleterious in cerebral malaria: A double-blind clinical trial in 100 comatose patients. $\mathrm{N}$ Engl J Med 1982;306:313-318.

58. Steketee RW, Nahlen BL, Parise ME, et al. The burden of malaria in pregnancy in malaria-endemic areas. Am J Trop Med Hyg 2001;64:28-35.

59. McGregor I. Epidemiology, malaria, and pregnancy. Am J Trop Med Hyg 1984;33:517.

60. Ahmed SM, Abd Al-Rhim SK, Mohamedani AA, et al. Malaria parasitemia during delivery. Saudi Med J 2002; 23:684-688.

61. Garner P, Gulmezoglu AM. Prevention versus treatment for malaria in pregnant women. Cochrane Database Syst Rev 2000:CD000169.

62. Steketee RW, Wirima JJ, Slutsker L, et al. Malaria prevention in pregnancy: The effects of treatment and chemoprophylaxis on placental malaria infection, low birth weight and fetal, infant and child survival. CDC-ARTS Publication 099-4048. Washington, DC: U.S. Dept of Health and Human Services; 1994.

63. Olliaro P, Cattani J, Wirth D. Malaria, the submerged disease. JAMA 1996;275:230.

64. Graves P, Gelband H. Vaccines for preventing malaria. Cochrane Database Syst Rev 2000:CD000129. 


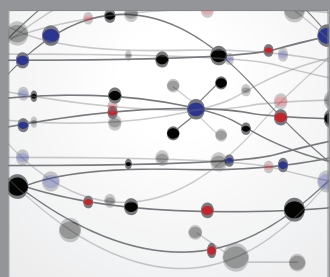

The Scientific World Journal
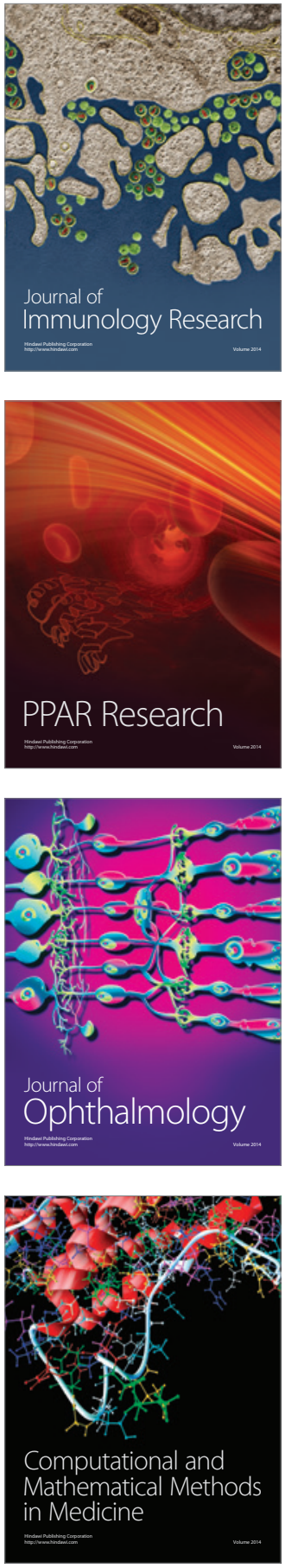

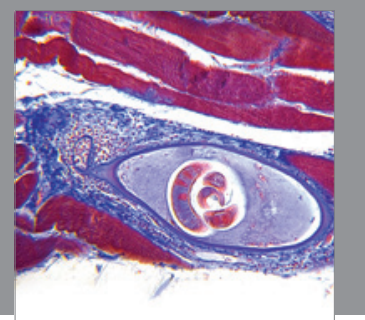

Gastroenterology

Research and Practice
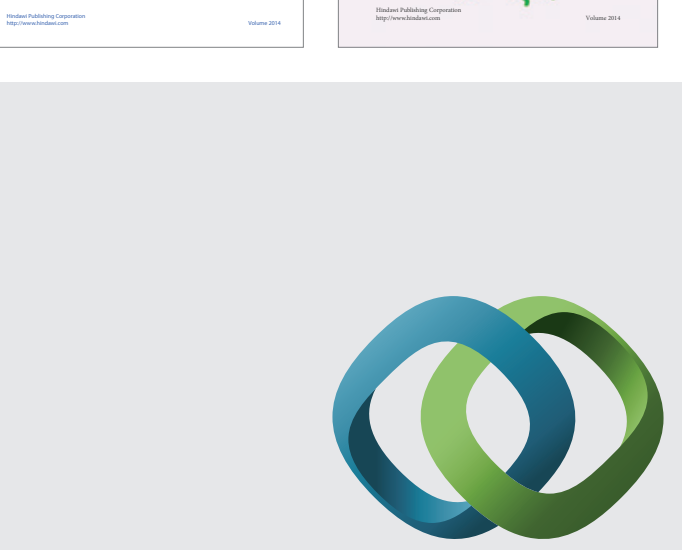

\section{Hindawi}

Submit your manuscripts at

http://www.hindawi.com
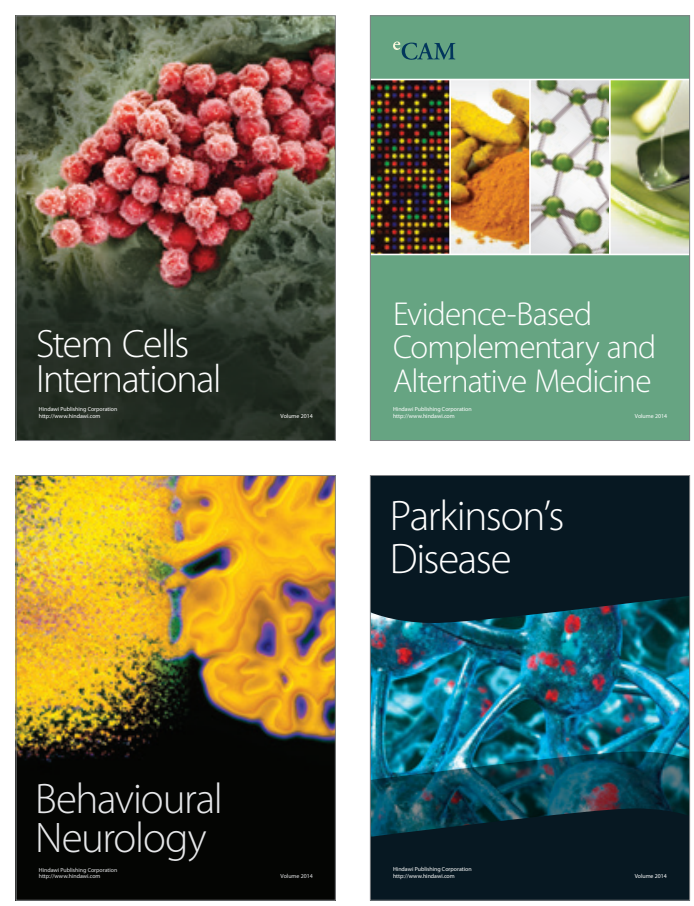

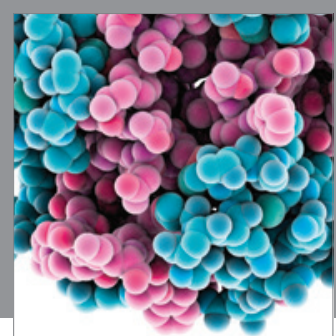

Journal of
Diabetes Research

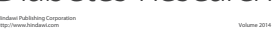

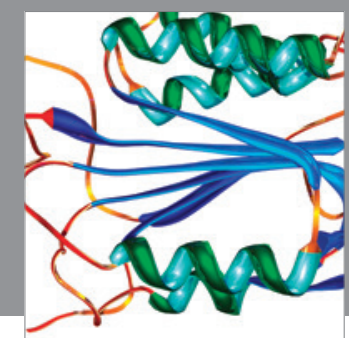

Disease Markers
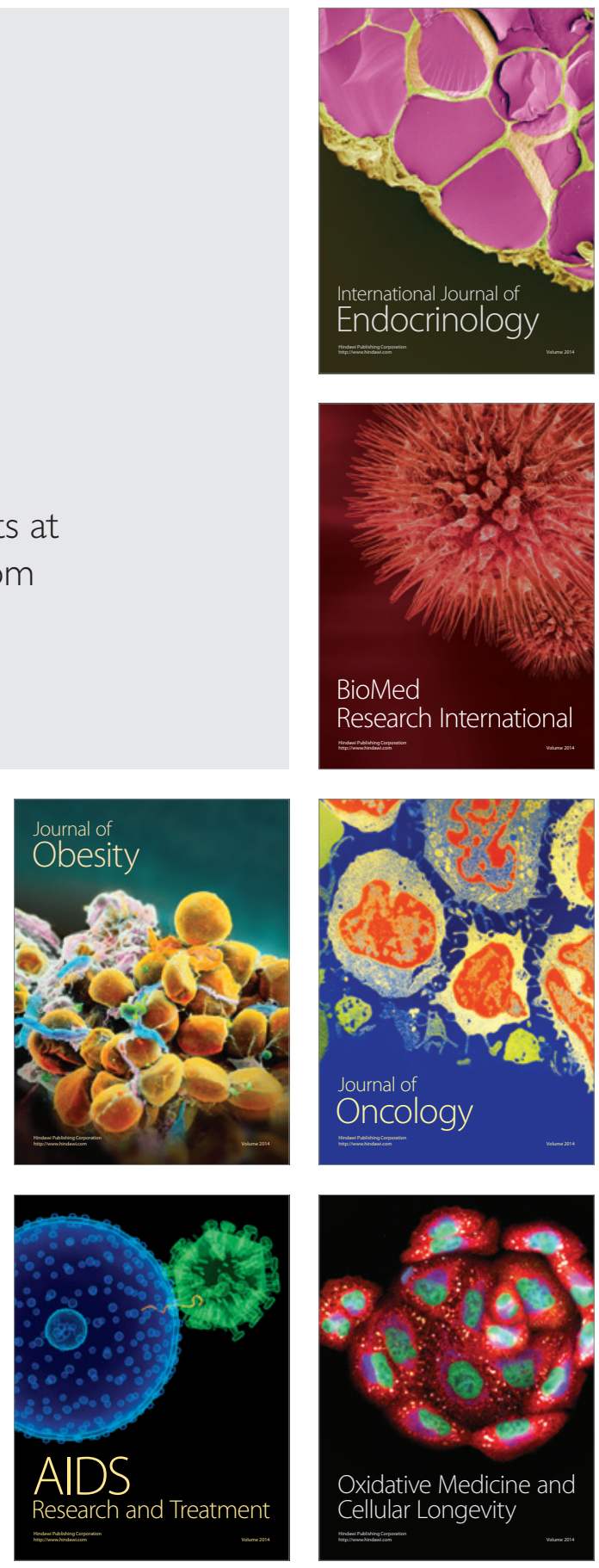